Ann. Biol. anim. Bioch. Biophy's., I964, 4 (4). 44I-444.

\title{
CONSOMMATION SPONTANÉE D'ENERGIE EN ALIMENTATION MIXTE ET SEPARÉE CHEZ LE PORC EN CROISSANCE
}

\author{
A. RÉRAT et Y. HENRY \\ avec la collaboration technique de E. ExgRavD et C. DAULOUder \\ Station de Recherches sur l'Élevage des Porcs \\ Centre national de Recherches sootechniques, Jouy-en-Josas (Seine-et-Oise).
}

Au cours d'une étude réalisée sur le Rat (HENRY et RÉRAT, sous presse), nous avons observé que lorsque ce dernier reçoit, en deux repas séparés, une quantité limitée de matières azotées, inférieure à celle correspondant à la vitesse de croissance maximum, et des éléments énergétiques à volonté, il ajuste spontanément sa consommation d'énergie à un niveau inférieur à celui qu'il se fixe en régime mixte, pour une même ingestion azotée ; cette réduction de la consommation d'énergie se traduit au niveau des tissus par une diminution sensible de l'adiposité, alors que la rétention azotée n'est pas modifiée. Une expérience a été entreprise dans les mêmes conditions sur le Porc, portant sur l'étude de la consommation spontanée d'énergie, selon qu'on administre à l'animal, en alimentation mixte ou séparée, la même quantité de matières azotées, l'énergie étant allouée à volonté.

\section{MATÉRIEL E'T MÉTHODES}

Deux lots de 9 porcs de race Large White, répartis par couples de même portée, de même sexe et d'un poids moyen initial de $25.3 \mathrm{~kg}$, sont soumis l'un à l'alimentation mixte, l'autre à l'alimentation séparée, jusqu'à $90 \mathrm{~kg}$ de poids vif. A cet effet, un animal de chaque couple reçoit ad libitum un régime mixte renfermant i 2 p. 100 de matières azotées sous forme de farine de poisson de Norvège. Au deuxième animal on distribue quotidiennement, dans deux compartiments séparés de l'auge, un régime protidique apportant la même quantité de matières azotées que celle ingérée la veille par son partenaire, et un régime protéiprive ad libitum. Les 3 régimes sont du type semi-synthétique et renferment, en dehors de la farine de poisson, les mêmes proportions d'huile d'arachide ( 5 p. Iоo), de sucre (3 p. 100), de cellulose de bois ( 5 p. 10o), de sels minéraux 
( 5 p. Ioo) et de mélange vitaminique ( 2 p. Ioo), le complément de la ration étant constitué par de l'amidon de maïs; leur valeur énergétique est donc sensiblement la même et de l'ordre de $\mathrm{r,2}$ U. F par kg. Ils sont distribués en granulés de $5 \mathrm{~mm}$ de diamètre; l'eau de boisson est fournie à volonté à laide d'abreuvoirs automatiques.

\section{RÉSULTATS E'T DISCUSSION}

L'examen des résultats de croissance et de consommation (tabl. I) fait apparaitre une grande similitude entre les deux lots, principalement au cours des deux périodes $40-60 \mathrm{~kg}$ et $60-90 \mathrm{~kg}$ de poids vif. Ainsi les écarts de croissance, s'ils sont en faveur du lot mixte, sont loin d'atteindre le seuil de signification; de la même façon, on n'observe aucune différence en ce qui concerne la consommation journalière de matière sèche et l'indice de consommation. Par contre, pendant la première phase de la croissance $(25-40 \mathrm{~kg})$ l'administration de repas séparés semble se traduire par un certain ralentissement de la croissance par rapport à l'alimentation mixte, tandis que la consommation de matière sèche est relativement moins importante. Il en résulte que le taux de matières azotées dans la ration sèche est plus élevé au début de la croissance dans le cas de l'alimentation séparée (I5,5 contre I 4,3 p. Ioo en régime mixte) ; de plus, il diminue régulièrement au cours des 3 périodes.

\section{TABLEAU I}

Résultats généraux de croissance et de consommation

\begin{tabular}{|c|c|c|c|c|c|c|c|c|c|}
\hline \multirow{2}{*}{ Púriode } & \multicolumn{3}{|c|}{$25-10 \mathrm{~kg}$} & \multicolumn{3}{|c|}{$40-60 \mathrm{kgr}$} & \multicolumn{3}{|c|}{$60-90 \mathrm{~kg}$} \\
\hline & Mixte & Séparé & $s \bar{x}(1)$ & Mixte & Séparé & $s \bar{x}\left({ }^{1}\right)$ & Mixte & Séparé & $s \bar{x}\left({ }^{1}\right)$ \\
\hline Gain moyen, $\mathrm{g} / \mathrm{j} \ldots$ & ${ }_{4} 95$ & 471 & 16 & 752 & 729 & 12 & 786 & 781 & 32 \\
\hline Protéines ingérées, $g / j$ & 179 & 180 & & $27 i$ & 283 & & 326 & 329 & \\
\hline Mat. sèche ingérée $\mathrm{kg} / \mathrm{j} \ldots \ldots$ & 1,25 & 1,17 & 0,03 & $1,9^{\prime} \mathrm{x}$ & $1,9 \div$ & 0,05 & 2,28 & 9,35 & 0,08 \\
\hline Protéines consommées p.100 & & & & & & & & & \\
\hline matière sc̀che $\ldots \ldots \ldots \ldots$ & 14,3 & 15,5 & 0,8 & 14,3 & $1 / 4,7$ & 0,7 & $1 t_{t}, 3$ & 14,0 & 0,4 \\
\hline Indice de consommation ( $\left.{ }^{2}\right)$ & 2,68 & 2,55 & 0,08 & $\because, 60$ & $\because, 69$ & 0,07 & 2,90 & 2,99 & 0,03 \\
\hline
\end{tabular}

(1) Ecart-type de la moyenne estimé au seuil 0,05 ; valeur de $t$ correspondante $=2,31$ pour 8 DL

(2) Mat. Sèche ingérée $(\mathrm{kg}) /$ gain de poids $(\mathrm{kg})$

En ce qui concerne la composition corporelle, on n'observe aucune influence significative du mode d'administration des éléments azotés et énergétiques. Ainsi les pourcentages de (jambon + longe) et de (bardière $\div$ panne) par rapport au poids net de la carcasse sont respectivement, en alimentation mixte 48,6 et 21,8 , et 
en alimentation séparée 49,0 et $2 I, 2$; les valeurs correspondantes de l'épaisseur du lard dorsal $\frac{\text { (Rein }+ \text { Dos) }}{2}$ sont respectivement $32,432,2 \mathrm{~mm}$.

Ce qui ressort le plus nettement de la présente étude, c'est que le Porc a tendance, au début de sa croissance, à consommer spontanément moins d'énergie par rapport à l'azote, au moment où son besoin azoté est le plus intense ; ce fait est en accord avec les observations de EvVARD (I9I5) et de LASsiter et al. (I955), qui opéraient sur des animaux en libre consommation, azotée et énergétique. Par ailleurs, au cours d'une étude précédente (RÉRAT et HENRY, I964), il a été observé que le taux de protéines de poisson qui provoque la vitesse de croissance maximum chez le Porc est de I 6 p. Ioo jusqu'à $45 \mathrm{~kg}$ de poids vif et de I 2 p. Ioo au-delà pour des régimes de même valeur énergétique que ceux utilisés dans 1'expérience présente. Etant donné que le régime mixte à I2 p. Ioo de protéines ne couvre pas complètement le besoin du porc pendant la première phase de la croissance, on peut penser que ce dernier en consomme le plus possible pour satisfaire son besoin azoté; pour cela il lui a fallu ingérer un excès d'énergie par rapport à l'azote. Par contre, lorsqu'il dispose de la même quantité d'azote, mais avec apport séparé d'énergie, il est en mesure de corriger un déséquilibre éventuel entre les fractions azotée et énergétique en réduisant sa consommation d'énergie par rapport à son ingestion d'azote, et sans pour cela que sa vitesse de croissance soit réduite d'une manière significative.

Si l'on rapproche ces résultats de ceux observés dans les mêmes conditions chez le Rat (HENRY et RÉRAT, sous presse), on peut penser que l'excès d'énergie qui est consommé par le Potc dans le cas du régime mixte se déposerait sous forme de lipides dans l'organisme, ce qui permettrait d'expliquer la différence dans le gain de poids. A partir de $40 \mathrm{~kg}$ de poids vif, au contraire, le besoin azoté est théoriquement couvert ; 1'apport azoté n'étant plus le facteur limitant de la croissance, il est logique de penser que les animaux se comportent de la mêtme façon en alimentation mixte et séparée, du point de vue de leur consommation spontanée d'énergie. C'est ainsi qu'à go $\mathrm{kg}$ de poids vif les performances sont sensiblement les mêmes et la composition corporelle n'est pratiquement pas modifiée.

Reçu pour publication en novembre 1964 .

\section{SUMMARY}

VOLUNTARY INTAKE OF ENERGY BY GROWING PIGS FED ON COMPLETE MIXED FEED OR SEPARATE INGREDIENTS

When pigs are fed, between 25 and $90 \mathrm{~kg}$ liveweight, to appetite on a complete feed containing i 2 p. Ioo fish protein, or are given the same amount of fish protein with energy allowed to appetite in separate feeds, there is little difference in performance as regards growth rate, intake of energy, or body composition. However, during the first phase of growth, from 25 to $40 \mathrm{~kg}$ liveweight, when the ration of protein is not sufficient to allow maximum growth, there seems to be an attempt to correct the imbalance between the protein and energy portions of the feed by reducing intake of energy in relation to that of protein, and this is the reason for the significant reduction in growth rate. 


\section{RÉFÉRENCES BIBIIOGRAPHIQUES}

Evvard J. M., 19r5. Is the appetite of swine a reliable indication of physiological needs? Iora Acad. Sci. 22, 375-403.

Henry Y., Rérat A., 1965. Consommation spontanée d'énergie en alimentation mixte ou séparée chez le Rat en croissance. Ann. Biol. anim. Biophys. (sous presse).

Lassiter J.W., Terril S. W., Becker D. E., Norton H. W., I955. Protein levels for pigs as studied by growth and self-selection. J. Anim. Sci. 14, 482-491.

Réat A., Henry Y., 1964. Etude du besoin azoté chez le Porc en croissance. I. Utilisation de la farine de poisson à trois taux différents. Ann. Zootech. 13, 5-34. 